\title{
Jahn-Teller Effects in a Vanadate-Stabilized Manganese-Oxo Cubane Water Oxidation Catalyst
}

\author{
Sebastian Mai, ${ }^{a, *}$ Marcus Holzer, ${ }^{a}$ Anastasia Andreeva, ${ }^{a}$ Leticia González ${ }^{a, *}$
}

\begin{abstract}
We report Jahn-Teller effects in different oxidation states of the water oxidation catalyst $\left[\left(\mathrm{Mn}_{4} \mathrm{O}_{4}\right)\left(\mathrm{V}_{4} \mathrm{O}_{13}\right)(\mathrm{OAc})_{3}\right]^{n-}$ and its activated form $\left[\left(\mathrm{Mn}_{4} \mathrm{O}_{4}\right)\left(\mathrm{V}_{4} \mathrm{O}_{13}\right)(\mathrm{OAc})_{2}\left(\mathrm{H}_{2} \mathrm{O}\right)(\mathrm{OH})\right]^{n-}$. Based on all combinatorially possible Jahn-Teller axis arrangements of the $\mathrm{Mn}^{\mathrm{III}}$ atoms, the energetically stable minima are identified. We also derive five heuristic rules that associate a particular energetic cost with certain structural features, like crossings of multiple Jahn-Teller axes, the location of Jahn-Teller axes, or the ligand that is involved in a Jahn-Teller axis. It is found that the different oxidation states seem to localize on different Mn centers, giving rise to clear Jahn-Teller distortions, unlike in previous crystallographic findings where an apparent valence delocalization was found. We conclude that the combination of cubane-vanadate bonds that are chemically inert, cubane-acetate/water bonds that can be activated through a Jahn-Teller axis, and low activation barriers for intramolecular rearrangement of the Jahn-Teller axes plays an important role in the reactivity of this and related compounds.
\end{abstract}

\section{Introduction}

In water splitting $\left(2 \mathrm{H}_{2} \mathrm{O} \rightarrow \mathrm{O}_{2}+2 \mathrm{H}_{2}\right)$, several molecular components are required, including a photosensitizer, a hydrogen evolution catalyst, and a water oxidation catalyst (WOC). The need for good WOCs has motivated an immense research effort in the last decades, ${ }^{[1]}$ given the many requirements WOCs needs to fulfill. These include high catalytic activity, stability, and sustainability-like being free of rare metals, having oxidatively robust ligands, supporting multiple oxidation states, enabling proton-coupled electron transfer (PCET), and others. ${ }^{[1-4]}$

One class of compounds able to catalyze the water oxidation reaction are manganese oxo clusters with a $\mathrm{Mn}_{4} \mathrm{O}_{4}$ cubane core. ${ }^{[5-9]}$ They are related to the reactive site of natural photosystem II, which contains the oxygen evolving complex that features an $\mathrm{Mn}_{4} \mathrm{CaO}_{5}$ cluster. Among other factors, it is consensus that this cluster is effective in catalyzing this reaction due to the availability of many oxidation states in the manganese atoms, ${ }^{[10]}$ where oxidation states $+\mathrm{III}$ and $+\mathrm{IV}$ are thought to play the central role. ${ }^{[11-13]}$

As a non-biological and simpler model to the oxygen evolving complex, the $\mathrm{Mn}_{4} \mathrm{O}_{4}$ cubanes were subject to significant research effort. The first such complex, with six diphenylphosphinate $\left(\mathrm{Mn}_{4} \mathrm{O}_{4} \mathrm{~L}_{6}\right.$, with $\left.\mathrm{L}=\mathrm{O}_{2} \mathrm{PPh}_{2}^{-}\right)$ligands, was reported by Dismukes and coworkers ${ }^{[5]}$ and further studied in the same group ${ }^{[14-17]}$ and by others. ${ }^{[18]}$ The structure of the compound showed no discernible Jahn-Teller (JT) distortions despite the formal presence of $\mathrm{Mn}^{\mathrm{III}}$ atoms. The oxidized species, $\mathrm{Mn}_{4} \mathrm{O}_{4} \mathrm{~L}_{6}^{+},{ }^{[14]}$ on the contrary, showed unequal bond lengths in the cubane structure. It was also shown ${ }^{[6]}$ that simply increasing the electron-donating abilities of the ligands can lead to the localization of the $\mathrm{Mn}^{\mathrm{III}}$ and $\mathrm{Mn}^{\mathrm{IV}}$ oxidation states, introducing JT distortions to the cubane structure. $\mathrm{Mn}_{4} \mathrm{O}_{4}$ cubane structures with localized valences and JT distortions were also reported with six bidentate dimethylarsenic acid ligands, ${ }^{[19]}$ or in heteroleptic compounds with diphenylphosphinate, acetate, and a hexadentate pyridine/alcohol ligand. ${ }^{[9,20,21]} \mathrm{JT}$ distortions were also found in manganese-oxo compounds like adamantane structures ${ }^{[22]}$ and others. ${ }^{[23]}$

${ }^{a}$ Dr. Sebastian Mai; Marcus Holzer, BSc.; Anastasia Andreeva, BSc.; Univ.-Prof. Dr. Leticia González, Institute of Theoretical Chemistry, Faculty of Chemistry, University of Vienna, Währinger Straße 17, 1090 Vienna, Austria. Email: sebastian.mai@univie.ac.at, leticia.gonzalez@univie.ac.at 

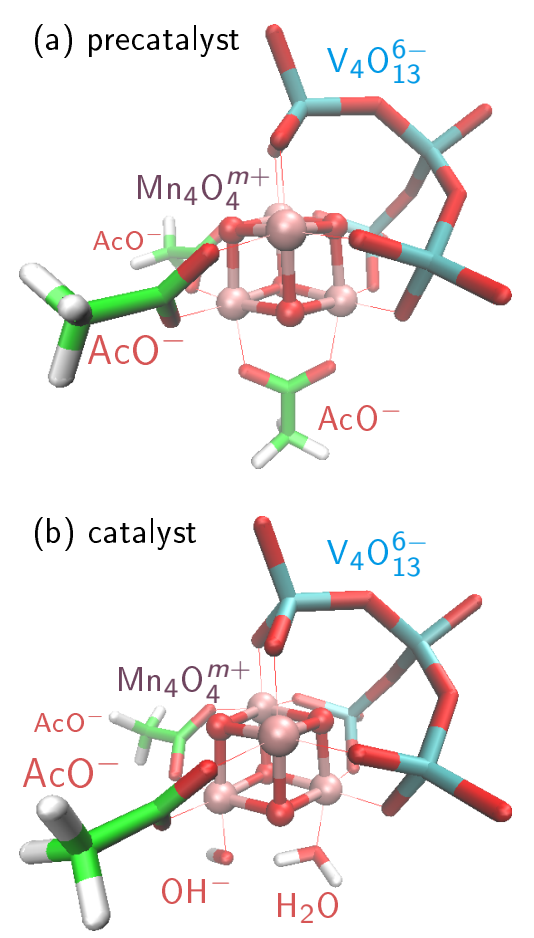

Figure 1: Depiction of the MnV WOC precatalyst with three acetate ligands (a) and the catalytically active form (b), where one of the acetates is exchanged for a water ligand and a hydroxide ligand. Mn atoms are colored pink, $\mathrm{V}$ teal, $\mathrm{O}$ red, $\mathrm{C}$ green, and $\mathrm{H}$ white.

A recent addition to this list of $\mathrm{Mn}_{4} \mathrm{O}_{4}$ cubanes is the polyoxometalate-stabilized compound ${ }^{[8]}\left[\left(\mathrm{Mn}_{4} \mathrm{O}_{4}\right)\left(\mathrm{V}_{4} \mathrm{O}_{13}\right)(\mathrm{OAc})_{3}\right]^{3-}$ (called "MnV WOC" in the following). Its three-dimensional molecular structure is depicted in Figure 1a. It was shown to effectively catalyze water oxidation in acetonitrile when coupled to a compatible photosensitizer, achieving a turn-over frequency of $3.6 \mathrm{~s}^{-1}$ and a turn-over number of approximately 12,000. ${ }^{[24]}$ Part of this success is probably due to the stabilizing effect of the tetravanadate ligand that is quite redox inactive. However, the activation mechanism and catalytic reaction cycle are still not well understood. Recent work ${ }^{[25]}$ has shown that the catalytically active structure (Figure $1 \mathrm{~b}$ ) is formed after two oxidation steps (producing a formal oxidation state of $\left[\mathrm{Mn}_{4}^{\mathrm{IV}}\right]$ ) and the exchange of one acetate ligand for a water molecule and a hydroxide ion. It was further observed that during this ligand exchange, an electron transfer between two Mn centers accompanied by a rearrangement of the JT axes contributed to significantly lowering the barrier for the rate-determining step. One can therefore conclude that the localization of $\mathrm{Mn}^{\mathrm{III}}$ and $\mathrm{Mn}^{\mathrm{IV}}$ oxidation states as well as the orientation of JT axes of the $\mathrm{Mn}^{\mathrm{III}}$ centers (see Figure 2) significantly influences the reactivity of the MnV WOC.

(a)

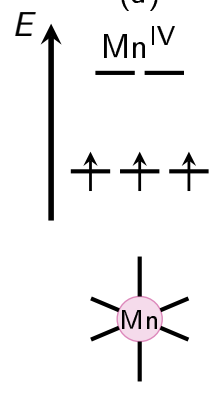

(b)

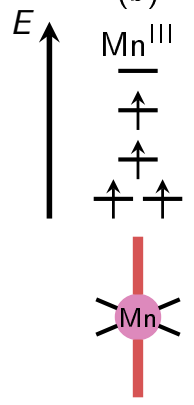

Figure 2: Orbital energy scheme for an ideal octahedrally coordinated $\mathrm{Mn}^{\mathrm{IV}}$ center (a) and a $\mathrm{Mn}^{\mathrm{III}}$ center with elongated octahedral coordination due to the JT effect that splits the two highest orbitals.

Here, we thus report on the influence of the JT effect on the structural stability of the MnV WOC for all oxidation 
Table 1: Considered oxidation states, corresponding labels, total charges, and total multiplicities.

\begin{tabular}{llcccc}
\hline $\begin{array}{l}\text { Label } \\
(\text { Kok })\end{array}$ & Label & $\begin{array}{c}n \\
\left(\mathrm{Mn}^{\mathrm{IV}}\right)\end{array}$ & $\begin{array}{c}n \\
\left(\mathrm{Mn}^{\mathrm{III}}\right)\end{array}$ & Charge & $\begin{array}{c}\text { Mult. } \\
2 S+1\end{array}$ \\
\hline$S_{3}$ & Mn4444 & 4 & 0 & -1 & 13 \\
$S_{2}$ & Mn3444 & 3 & 1 & -2 & 14 \\
$S_{1}$ & Mn3344 & 2 & 2 & -3 & 15 \\
$S_{0}$ & Mn3334 & 1 & 3 & -4 & 16 \\
$S_{-1}$ & Mn3333 & 0 & 4 & -5 & 17 \\
\hline
\end{tabular}

states between $\left[\mathrm{Mn}_{4}^{\mathrm{IV}}\right]$ and $\left[\mathrm{Mn}_{4}^{\mathrm{III}}\right]$. We consider systematically all possible combinations of location of the $\mathrm{Mn}^{\mathrm{III}}$ atoms and all JT axis orientations within the point group symmetry of the molecule, in order to find the preferred JT axis arrangements. We also investigate the interactions between the different JT orientations and the effects of substituting one acetate ligand by a water molecule and a hydroxide ion, as is observed in the activated catalyst. Furthermore, we revisit the question whether the oxidation states in the MnV WOC are localized or delocalized, by comparing our findings to previously published ${ }^{[8]} \mathrm{X}$-ray structures. This information is condensed into heuristic rules that allow the prediction of preferred JT axis arrangements in this complex (e.g., during catalyst activation or catalytic cycle) and might also inspire more general work about JT effects in metal-oxo cubanes.

\section{Methods}

\subsection{Notation}

We distinguish two species of the MnV WOC. The first is the unactivated precatalyst, ${ }^{[8]}$ which consists of the $\mathrm{Mn}_{4} \mathrm{O}_{4}$ cube, the $\mathrm{V}_{4} \mathrm{O}_{13}$ vanadate ligand, and three acetate ligands, which we will label "precatalyst" in the following. The second species is the activated catalyst, ${ }^{[25]}$ which is obtained from the precatalyst by release of one acetate ligand and attachment of one $\mathrm{H}_{2} \mathrm{O}$ ligand and one $\mathrm{OH}^{-}$ligand. This species will be referred to as "catalyst" below. Both structures are shown in Figure 1. Note that the central proton of the catalyst can easily move between the $\mathrm{H}_{2} \mathrm{O}$ and $\mathrm{OH}^{-}$ligands; thus, our investigations will include both tautomers.

Table 1 presents the investigated oxidation states. Note that for both precatalyst and catalyst the total charge of the ligands is identical, and therefore the table is valid for both species. The Kok-analogous labels, ${ }^{[10]}$ where $S_{3}$ corresponds to an $\left[\mathrm{Mn}_{4}^{\mathrm{IV}}\right]$ oxidation state and the $S_{-1}$ to an $\left[\mathrm{Mn}_{4}^{\mathrm{III}}\right]$ oxidation state, are also included. The $S_{-1}$ state was not part of the originally proposed Kok cycle, ${ }^{[10]}$ but such "super-reduced" states are nowadays discussed for the oxygen evolving complex ${ }^{[13]}$ and might also be of relevance in the MnV WOC. The simpler labels, like "Mn4444", clearly specify the oxidation states of the different atoms and therefore will be used below.

A thorough discussion of the JT distortions of the molecule requires that we define unique labels for each JT axis and all $\mathrm{Mn}$ atoms. Figure 3 shows the central cube structure of the precatalyst and activated catalyst. It also introduces the atom labels $A, B, C$, and $D$. Here, atom $A$ is distinguished from the others by the fact that it is not bonded directly to the vanadate ligand. We refer to this atom $A$ as the "apical" Mn atom (as will be discussed below, this atom behaves significantly different than the three other $\mathrm{Mn}$ atoms). The $\mathrm{O}$ atom opposite to $A$ is analogously referred to as the apical $\mathrm{O}$ atom. The other $\mathrm{Mn}$ and $\mathrm{O}$ atoms are referred to as "non-apical".

As can be discerned in Figure 3, the precatalyst with three equal acetate ligands exhibits $C_{3 v}$ symmetry (in the absence of JT distortions). The rotation axis passes through the apical $\mathrm{Mn}$ and $\mathrm{O}$ atoms, whereas each of the three mirror planes passes through these two atoms and one of atoms $B / C / D$. On the contrary, the activated catalyst has approximate $C_{s}$ symmetry, with the only mirror plane passing through the apical $\mathrm{Mn}$ and $\mathrm{O}$ atoms, atom $B$, and the $\mathrm{O}$ atoms of water and hydroxide. Note that in principle the positions of the $\mathrm{H}_{2} \mathrm{O} / \mathrm{OH}^{-}$protons can break this symmetry, but for the purpose of labeling the different JT distortions, we will ignore these protons. While we consider symmetry to identify all non-equivalent JT distortions, we did not employ explicit symmetry in the computations.

With regard to the JT distortions, each of the four Mn atoms can be in exactly one of four different states, either 


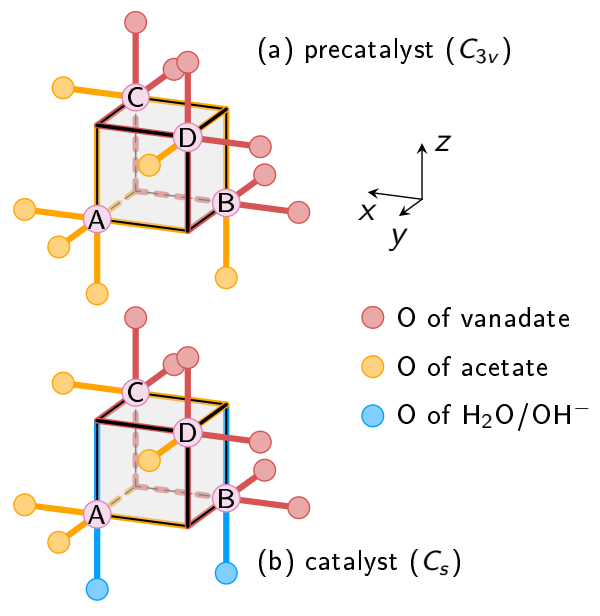

Figure 3: Schematic depictions of the central cube structure of the MnV catalyst and atom labels. (a) The precatalyst with three equivalent acetate ligands. (b) Activated catalyst with two acetate ligands and one pair of $\mathrm{H}_{2} \mathrm{O} / \mathrm{OH}^{-}$ligands. The orientation of the coordinate system is also depicted.

being an $\mathrm{Mn}^{\mathrm{IV}}$ atom without a JT axis, or being an $\mathrm{Mn}^{\mathrm{III}}$ atom with a JT axis in $x, y$, or $z$ direction. Hence, we build the labels for the different JT orientations from the characters "4", "x", "y”, and "z". For example, the label "z444" indicates that atom $A$ is a $\mathrm{Mn}^{\mathrm{III}}$ with JT axis in $z$ direction, and atoms $B, C$, and $D$ are $\mathrm{Mn}$ atoms with oxidation state 4 , i.e., $\mathrm{Mn}{ }^{\mathrm{IV}}$ atoms. Additionally, for the catalyst, we add an "O" or " $\mathrm{H}$ " prefix to the label, where "O" indicates that the $\mathrm{OH}^{-}$ligand is attached to atom $A$ and " $\mathrm{H}$ " indicates that $\mathrm{H}_{2} \mathrm{O}$ is attached to atom $A$. For example, the structure shown in Figure $1 \mathrm{~b}$ would be labeled "O4444".

\subsection{Optimization strategy}

As we aim at finding how the different JT axes of the two molecules are arranged in all stable local minima, we generate starting geometries for all possible, symmetry-nonequivalent JT distortions, which are then optimized. The starting geometries were produced from constrained optimizations, where the JT distortions were enforced by constraints. Mn-O bonds involved in the JT distortions were constrained to $2.10-2.30 \AA$, which is significantly longer than the $\mathrm{Mn}-\mathrm{O}$ bonds of $\mathrm{Mn}^{\mathrm{IV}}$ and helps to converge the initial wave function to the correct localization. These preoptimizations were carried out with loose optimization criteria and with smaller basis sets, as detailed below.

Subsequently, the preoptimized geometries were taken as initial geometries for unconstrained optimizations. The JT distortions of the resulting geometries were classified according to the involved 24 bond lengths (12 in the cube and 12 towards the ligands). For all distinct geometries, the optimization was followed by a frequency calculation to confirm the minimum and to compute the free energy of the minima including vibrational corrections. If the same geometry was reached in multiple optimizations from different preoptimized structures, then only one frequency calculation was performed.

\subsection{Electronic structure}

The preoptimizations were carried out with the ORCA 4.2.1 package. ${ }^{26,27]}$ We used the BP86 functional ${ }^{[28,29]}$ and the ZORA-def2-SVP ${ }^{[30,31]}$ basis set, where the zeroth order regular approximation (ZORA) ${ }^{[31-33]}$ was used to incorporate scalar relativistic effects. The calculations also employed the D3 dispersion correction ${ }^{[34]}$ and the C-PCM implicit solvation model ${ }^{[35]}$ to describe the acetontrile solvent used experimentally. ${ }^{[8]}$

The final optimizations and frequency calculations were performed with Gaussian 16. ${ }^{[36]}$ We employed the BP86 functional, the def2-SVP ${ }^{[30]}$ basis set for $\mathrm{C}$ and $\mathrm{H}$, and the larger def2-ZTVP ${ }^{[30]}$ basis set for Mn, V, and O. The GD3 dispersion correction ${ }^{[37]}$ was included. The effect of the solvent was described through the IEFPCM formalism. ${ }^{\text {[38] }}$ 
The functional was chosen based on several recommendations from literature ${ }^{[12,13]}$ that showed that the dispersioncorrected BP86 functional can produce reasonable geometries for multi-nuclear Mn complexes. We note that throughout the study, we have adhered to using unrestricted Kohn-Sham calculations assuming a high-spin configuration. Preliminary broken-symmetry calculations indicated that the complex is likely to be in a low-spin configuration, but that the energetic stabilization (about $1.3 \mathrm{kcal} / \mathrm{mol}$ for precatalyst Mn3344) from the broken-symmetry calculation is virtually independent of the geometry. Hence, we assume that our findings (relative energies, barrier heights) are not affected by using the computationally more efficient high-spin approach, as has been done previously on studies of the oxygen-evolving complex. ${ }^{[39]}$

We note that several of the Gaussian 16 optimizations showed convergence problems close to the minimum. These were solved by trying various SCF and geometry optimization schemes, with the minimum confirmed after convergence by a frequency calculation.

We performed additional calculations to compute potential energy scans for transitions between different minima of the precatalyst in the Mn3344 oxidation state. For these calculations, transition states were obtained with the climbingimage nudged elastic band method ${ }^{[40]}$ implemented in ORCA 4.2 .1 and a subsequent transition state optimization. The scans were obtained from linear interpolation in Cartesian coordinates, and for some scans the climbing image was used because the transition state optimization was not successful.

\subsection{Generation of the symmetry-inequivalent starting geometries}

In principle, the number of possible JT axis configurations can be computed in a combinatorial way. As there are four $\mathrm{Mn}$ atoms and each can be in any of four states $\left(\mathrm{Mn}^{\mathrm{IV}}\right.$ with no axis or $\mathrm{Mn}^{\mathrm{III}}$ with either $x, y$, or $z$ axis), there are in total $4^{4}=256$ possible JT arrangements for each molecule. This implies that, for the precatalyst and the catalyst (both tautomers), a total of $3 \times 256=768$ possible arrangements need to be considered. Molecular symmetry allows significantly reducing this large number of structures to be optimized. As the precatalyst exhibits $C_{3 v}$ symmetry, the JT arrangements are up to 6-fold degenerate. The catalyst only exhibits $C_{s}$ symmetry, giving many arrangements that are two-fold degenerate.

For example, for Mn3444, there is a threefold degenerate arrangement where the only JT axis is localized on the apical Mn atom (labeled z444, y444, and $\mathrm{x} 444$ ), a threefold degenerate arrangement where the JT axis is on $B / C / D$ and points towards an acetate $(4 \mathrm{z} 44,44 \mathrm{x} 4,444 \mathrm{y})$, and a sixfold degenerate arrangement with the JT axis on $B / C / D$ pointing towards the vanadate $(4 \mathrm{x} 44,4 \mathrm{y} 44,44 \mathrm{y} 4,44 \mathrm{z} 4,444 \mathrm{x}, 444 \mathrm{z})$. These 12 possibilities are shown in Figure S1 in the Supporting Information (SI). The entire list of all symmetry equivalences is given in Tables S1 and S2 in the SI.

For the precatalyst, considering symmetry there is a total of 52 symmetry-inequivalent JT arrangements. This includes 1 arrangement for Mn4444, 3 arrangements for Mn3444, 11 for Mn3344, 22 for Mn3334, and 15 for Mn3333. These 52 arrangements are presented in Figure S2. For each of the two tautomers of the catalyst, there are 136 symmetryinequivalent JT arrangements (1 for Mn4444, 7 for Mn3444, 29 for Mn3344, 57 for Mn3334, 42 for Mn3333). In order to make this still large number more manageable, based on the results from the precatalyst (see below), we decided to omit the optimization of all JT arrangements of Mn3334 and Mn3333 with JT axes pointing towards the vanadate ligand. This reduces the number of JT arrangements for Mn3334 to only 6 and for Mn3333 to only 2, giving a total of 45 investigated JT arrangements for each tautomer. These JT arrangements for all oxidation states of the catalyst are shown in Figures S3 $\left(\mathrm{OH}^{-}\right.$on apical position) and $\mathrm{S} 4\left(\mathrm{H}_{2} \mathrm{O}\right.$ on apical position).

In total, we considered 142 starting geometries including both molecules, all tautomers, and all oxidation states.

\section{Results and discussion}

\subsection{Jahn-Teller axis arrangements}

During the pre-optimizations and full optimizations, we monitored the convergence to the different minima through the averages of the bond lengths of all the 12 coordination axes of the four manganese atoms. The full set of average 
(a) precatalyst

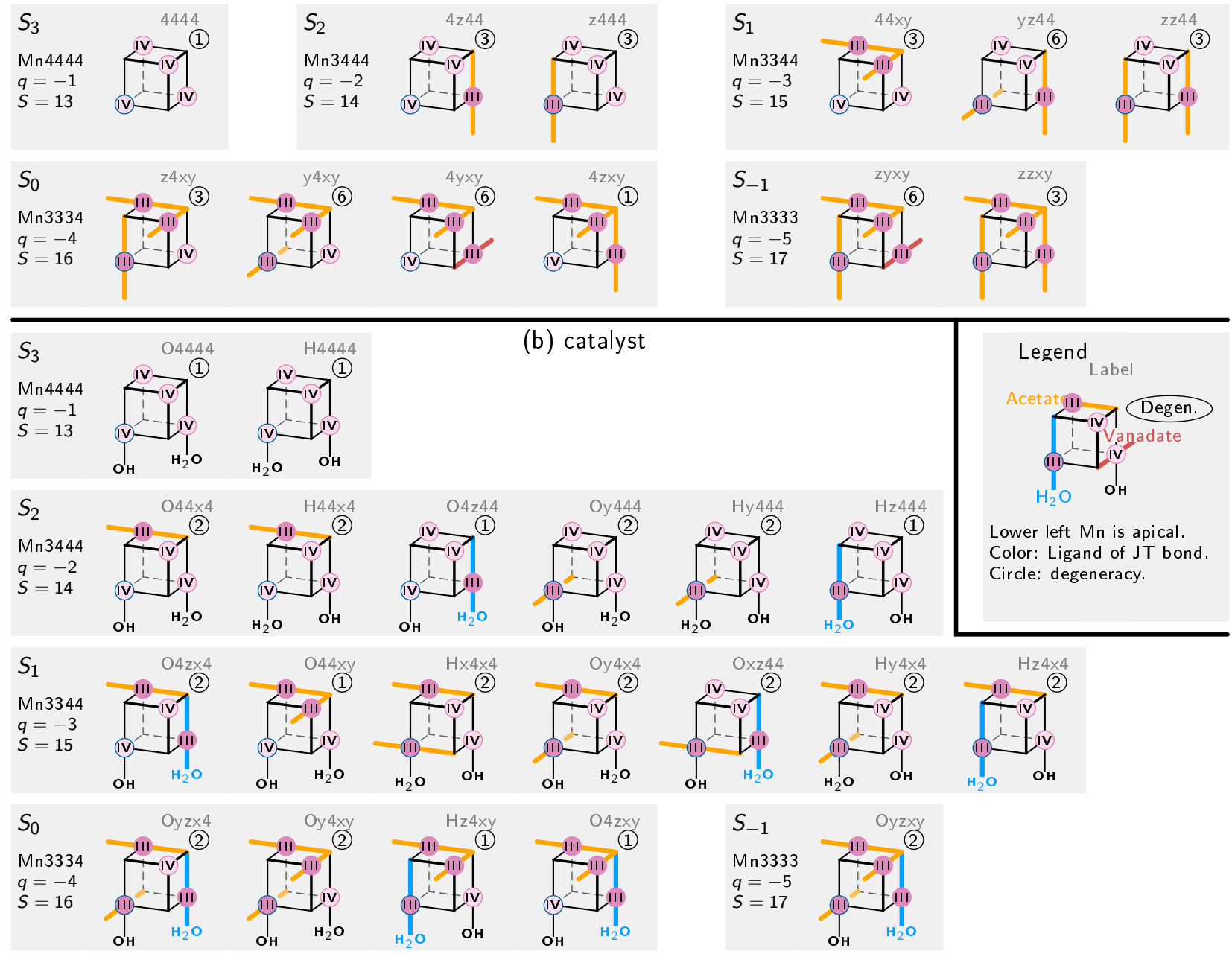

Figure 4: Overview over all JT axis arrangements of the precatalyst and the catalyst that are stable minima. Each block corresponds to one overall oxidation state $\left(S_{3}, S_{2}, \ldots\right.$; indicated by charge $q$ and spin $\left.S\right)$. Each cube is oriented in the same way, with the apical $\mathrm{Mn}$ atom in the lower-left corner. JT axes are indicated by colored, thick lines, with the color indicating the type of bonded ligand. The degeneracy of the arrangement and a label are also given.

bond lengths after pre-optimization and after full optimization is given in Tables S3, S4, and S5 in the SI. Using these average bond lengths, one can automatically identify non-JT and JT axes in the optimized structures. Non-JT axes are consistently in the $1.88 \AA-1.97 \AA$ range, whereas JT axes showed an average bond length of $2.17-2.32 \AA$.

The 142 optimizations lead to 32 different local minima, collected in Figure 4 (see also Figures S2, S3, and S4). The Cartesian coordinates of all 32 minima are collected in a supplementary file in XYZ format. For the precatalyst, there are 12 stable minima (Figure 4a). For Mn4444, the optimization lead to a fully $C_{3 v}$ symmetric geometry. For Mn3444, we found two different minima, one where the JT axis is located on a non-apical Mn (4z44) and one where the JT axis is located on the apical Mn atom (z444). All attempts to optimize a structure with the JT axis pointing towards the vanadate ligand failed, indicating that such JT axes are energetically unfavorable and lead barrierlessly to minima with the JT axis towards an acetate. Likewise, for Mn3344 we did not observe a stable minimum with a vanadate JT axis. Three different minima with two acetate JT axes are obtained, one where the JT axes intersect at the apical $\mathrm{O}$ atom, and two where one JT axis is on the apical Mn atom. These motifs-intersecting JT axes and a JT axis on the apical Mn-are also found in the Mn3334 and Mn3333 oxidation states. However, here we also find few stable minimum with one vanadate JT axis.

For the catalyst, the findings are slightly more diverse due to the larger number of different ligands. For Mn4444, we obtained two stable minima that only differ in the position of the water proton. For Mn3444, a total of six structures was found, where all exhibit JT axes towards either an acetate or the $\mathrm{H}_{2} \mathrm{O}$ ligand. No structures with the JT axis to the 
Table 2: Free energy differences between the different stable minima of the five oxidation states of the precatalyst.

\begin{tabular}{llccc}
\hline Label & Conf. & $\begin{array}{c}\Delta G \\
(\mathrm{kcal} / \mathrm{mol})\end{array}$ & $\begin{array}{c}\text { Degen- } \\
\text { eracy }\end{array}$ & $\begin{array}{c}\text { Population } \\
(300 \mathrm{~K})\end{array}$ \\
\hline Mn4444 & 4444 & 0.0 & 1 & $100 \%$ \\
\hline Mn3444 & $4 \mathrm{z} 44$ & 0.0 & 3 & $100 \%$ \\
& z444 & 9.7 & 3 & $0 \%$ \\
\hline Mn3344 & $44 x y$ & 0.0 & 3 & $99.9 \%$ \\
& yz44 & 3.9 & 6 & $0.1 \%$ \\
& zz44 & 4.4 & 3 & $0 \%$ \\
\hline Mn3334 & z4xy & 0.0 & 3 & $35 \%$ \\
& y4xy & 0.05 & 6 & $64 \%$ \\
& $4 y x y$ & 2.2 & 6 & $1 \%$ \\
& $4 z x y$ & 3.2 & 1 & $0 \%$ \\
\hline Mn3333 & zyxy & 0.0 & 6 & $100 \%$ \\
& zzxy & 5.1 & 3 & $0 \%$ \\
\hline
\end{tabular}

vanadate or the $\mathrm{OH}^{-}$were found to be stable. For Mn3344, seven different structures were found, out of the nine possible structures with JT axes to acetate or $\mathrm{H}_{2} \mathrm{O}$; again, $\mathrm{JT}$ axes to vanadate or $\mathrm{OH}^{-}$were not stable. For Mn3334 and Mn3333 of the catalyst, we only used starting structures without vanadate JT axes and found only four plus one minima.

Generally speaking, these findings strongly indicate that there are certain JT axis configurations that are energetically unfavorable, like JT axis pointing to the vanadate oxygens or towards the $\mathrm{OH}^{-}$ligand.

\subsection{Relative energies in the precatalyst}

The relative energies, degeneracies, and Boltzmann populations of the 12 stable minima for the precatalyst are given in Table 2. As can be seen, the energies of the different minima and the corresponding Boltzmann distributions differ substantially. For the precatalyst, in all oxidation states except Mn3334 there is only one minimum that is thermally populated at room temperature.

More interestingly, based on the results in Tables 2 and 3, we can find several heuristic rules that can be used to understand the different energies of the minima in a general fashion. The first rule can be obtained by comparing the energies of the two minima for the precatalyst in the Mn3444 oxidation state $\left(S_{2}\right)$. Here, the minimum with the JT axis at the apical $\mathrm{Mn}$ atom $(\mathrm{z} 444)$ is almost $10 \mathrm{kcal} / \mathrm{mol}$ less stable than the minimum with the JT axis at the non-apical Mn atom (4z44). This very large difference is not due to the ligand located along the JT axis, as in both cases this is an acetate $\mathrm{O}$ atom. Instead, it can be assumed that the ligands orthogonal to the JT axis are responsible for the difference in energy. The JT axis at the non-apical atom is neighbored by two vanadate $\mathrm{O}$ atoms, whereas the JT axis on the apical atom is flanked by two acetate $\mathrm{O}$ atoms. It appears that the vanadate $\mathrm{O}$ atoms produce a stronger ligand field splitting at the non-apical Mn atoms, which leads to a stronger stabilization of the $d_{z^{2}}$ orbital and makes a JT axis on these atoms energetically more favorable.

For the second rule, we direct our attention to the three minima for the precatalyst in the Mn3344 oxidation state $\left(S_{1}\right)$. The lowest-energy minimum (44xy) features two non-apical JT axes that point towards acetate $\mathrm{O}$ atoms and cross at the apical $\mathrm{O}$ atom. The two other minima, about $4 \mathrm{kcal} / \mathrm{mol}$ above the lowest-energy minimum, both feature an energetically unfavorable apical JT axis (yz44 and zz44). If we assume that the energetic cost of an apical JT axis is about $10 \mathrm{kcal} / \mathrm{mol}$, then the $4 \mathrm{kcal} / \mathrm{mol}$ energy difference could be explained by a second rule that the crossing of two JT axes entails an extra energetic cost of approximately $6 \mathrm{kcal} / \mathrm{mol}$. One possible explanation for this behaviour might be the unfavorable electrostatic repulsion due to a high electron density at the apical $\mathrm{O}$ atom from multiple occupied $d_{z^{2}}$ orbitals pointing in its direction. As the energies of the yz44 and zz44 minima are roughly identical, we can further conclude that a skew or parallel arrangement of two JT axes does not notably affect the energy (although parallel JT axes seem to be slightly less favorable).

We can now apply these two rules to the four optimized minima of the Mn3334 oxidation state $\left(S_{0}\right)$. The $\mathrm{z} 4 \mathrm{xy}$ and y4xy minima both feature an apical JT axis as well as a two-axes crossing, and differ only in the relative direction of the 
JT axes. Hence, they should have similar energies, in good agreement with the $0.05 \mathrm{kcal} / \mathrm{mol}$ difference obtained in the calculations. The two other minima (4yxy and 4zxy) do not have an unfavorable apical JT axis but nevertheless a higher energy. Hence, the presence of a JT axis towards a vanadate $\mathrm{O}$ (in 4yxy) and a three-axes crossing (in 4zxy) appear to entail an additional cost. We can thus establish rule three, that a vanadate JT axis costs about $12 \mathrm{kcal} / \mathrm{mol}$. Likewise, in rule four we state that a three-axes crossing costs $13 \mathrm{kcal} / \mathrm{mol}$ more than a two-axes crossing-19 $\mathrm{kcal} / \mathrm{mol}$ in total, which is almost exactly three times as much as a two-axes crossing. The energetic cost of the vanadate JT axes can be rationalized by the fact that it is neighbored by an acetate $\mathrm{O}$ atom, which is a weak ligand (weaker than a vanadate $\mathrm{O}$ atom) and leads to a weak ligand field splitting.

For the Mn3333 state $\left(S_{-1}\right)$, in principle these four rules should be sufficient to predict the energetics, but the predicted energy difference ( $1 \mathrm{kcal} / \mathrm{mol}$ difference, as zyxy has a vanadate JT axis and zzxy has a three-axes crossing) does not agree fully with the computed $5 \mathrm{kcal} / \mathrm{mol}$ energy difference. However, inspection of the optimized geometries shows that zyxy actually dissociates one acetate $\mathrm{O}$ atom, leading to a five-coordinated $\mathrm{Mn}$ atom. As it is difficult to estimate the possible effect of filling this vacancy with another solvent molecule, we prefer to not consider this minimum further.

With the four proposed rules, we can now estimate the energetic cost of those structures that turned out to not be stable minima. A table with all symmetry-inequivalent JT arrangements, their energy predicted by these rules, and the computed energies for comparison are given in Table S6 in the SI. For example, for Mn3444 there is one JT arrangement that did not lead to a stable minimum, containing one JT axis towards a vanadate. Hence, according to rule three, this hypothetical structure would have an energy of about $12 \mathrm{kcal} / \mathrm{mol}$, higher than both of the stable minima for this oxidation state $(0 \mathrm{kcal} / \mathrm{mol}$ for $4 \mathrm{z} 44,10 \mathrm{kcal} / \mathrm{mol}$ for $\mathrm{z} 444)$. For Mn3344, out of the 11 possible JT arrangements only 3 were found to be stable, with predicted energies of about $6 \mathrm{kcal} / \mathrm{mol}$ (44xy) and $10 \mathrm{kcal} / \mathrm{mol}$ (yz44 and zz44). The four rules successfully predict that the 8 other hypothetical JT arrangements (see Figure S2) are all higher in energy (the structures $44 \mathrm{xx}$ and $44 \mathrm{xz}$ with predicted energies of about $12 \mathrm{kcal} / \mathrm{mol}$ would be the next lowest). The rules also work similarly for Mn3334, where other JT arrangements would be at least $3 \mathrm{kcal} / \mathrm{mol}$ higher in energy than any of the four stable minima.

These rules explain why vanadate JT axes-although energetically unfavorable-can appear for the low oxidation states: Those states are so overcrowded with JT axes that a high energetic cost comes from axes crossings or an apical JT axis. Hence, it might be energetically more favorable to accept a vanadate JT axis in order to get rid of three-axes crossings or an apical JT axis that are also energetically unfavorable. On the contrary, for the high oxidation states, JT axis arrangements without vanadate axes are energetically clearly favored.

\subsection{Relative energies in the catalyst}

The analogous results on the relative energies in the catalyst are compiled in Table 3. As can be see, in the activated complex, in most oxidation states the calculations predict a thermal mixture of distinguishable minima. In principle, the data in the table is consistent with the discussion above for the precatalyst. However, in order to explain all energies, we need to adjust some of the energetic costs and add at least one more rule.

The free energies given in Table 3 for the Mn4444 $\left(S_{3}\right)$ state show that in the absence of any JT axes, the position of the proton (O4444 versus H4444, see also Figure $4 \mathrm{~b}$ ) does not significantly affect the energy. The small energy difference of $0.2 \mathrm{kcal} / \mathrm{mol}$ indicates that probably no specific rule is necessary here. However, we note that all lowest-energy minima for all oxidation states have the hydroxide ligand at the apical Mn atom and the water ligand at the non-apical one.

For Mn3444 $\left(S_{2}\right)$, we observe again a very small energetic difference due to the position of the proton in two cases (O44x4 versus H44x4, Oy444 versus Hy444). We also see that the first rule found above-that an apical JT axis has a significant energetic cost-is nicely reproduced here. However, assessing the energies of the affected arrangements (Oy444, Hy444, Hz444) shows that for the catalyst the cost of the apical JT axis is slightly lower at about $7.5 \mathrm{kcal} / \mathrm{mol}$ (compared to $10 \mathrm{kcal} / \mathrm{mol}$ in the precatalyst). This is probably due to the different ligand field of the water/hydroxide ligands, compared to a third acetate ligand. For the same reason, we observe a differentiation between the non-apical, non-vanadate JT axes. As can be seen from comparing O44x4 and O4z44, an acetate JT axis and a water JT axis are slightly different $(0.5 \mathrm{kcal} / \mathrm{mol})$ in energy, with the acatete JT axis being slightly more favorable. Additional information 
Table 3: Free energy differences between the different stable minima of the five oxidation states of the catalyst.

\begin{tabular}{llccc}
\hline Label & Conf. & $\begin{array}{c}\Delta G \\
(\mathrm{kcal} / \mathrm{mol})\end{array}$ & $\begin{array}{c}\text { Degen- } \\
\text { eracy }\end{array}$ & $\begin{array}{c}\text { Population } \\
(300 \mathrm{~K})\end{array}$ \\
\hline Mn4444 & O4444 & 0.00 & 1 & $61.2 \%$ \\
& H4444 & 0.24 & 1 & $38.8 \%$ \\
\hline Mn3444 & O44x4 & 0.00 & 2 & $59.3 \%$ \\
& H44x4 & 0.37 & 2 & $29.4 \%$ \\
& O4z44 & 0.51 & 1 & $11.3 \%$ \\
& Oy444 & 7.46 & 2 & $0 \%$ \\
& Hy444 & 7.67 & 2 & $0 \%$ \\
& Hz444 & 7.70 & 1 & $0 \%$ \\
\hline Mn3344 & O4zx4 & 0.00 & 2 & $73.0 \%$ \\
& O44xy & 0.22 & 1 & $24.0 \%$ \\
& Hx4x4 & 2.00 & 2 & $1.7 \%$ \\
& Oy4x4 & 2.63 & 2 & $0.5 \%$ \\
& Oxz44 & 2.67 & 2 & $0.5 \%$ \\
& Hy4x4 & 2.81 & 2 & $0.4 \%$ \\
& Hz4x4 & 4.21 & 2 & $0 \%$ \\
\hline Mn3334 & Oyzx4 & 0.00 & 2 & $94.0 \%$ \\
& Oy4xy & 1.52 & 2 & $5.3 \%$ \\
& Hz4xy & 2.22 & 1 & $0.7 \%$ \\
& O4zxy & 4.20 & 1 & $0 \%$ \\
\hline Mn3333 & Oyzxy & 0.0 & 2 & $100 \%$ \\
\hline
\end{tabular}

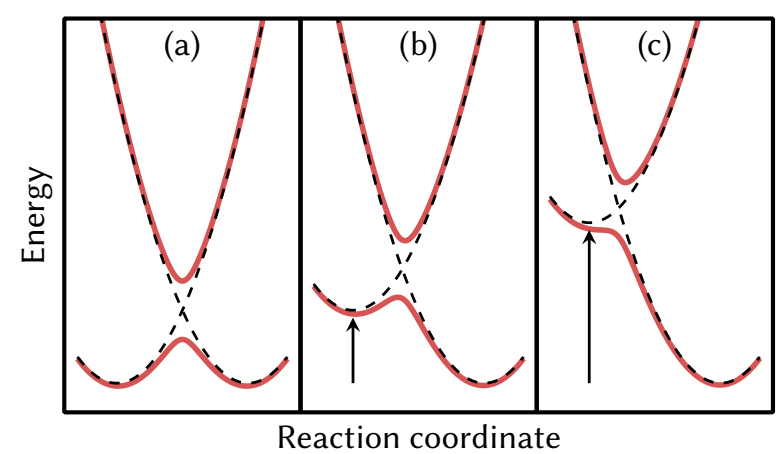

Figure 5: Simple two-state diabatic model explaining how energy shifts of a minimum can reduce and remove a transition barrier towards a lower state. In (a), both diabatic minima (dashed) have the same energy and the barrier arises from electronic coupling of the two diabats. In (b) and (c), the left minimum is shifted to higher energy, such that in (c) the left diabat does not produce a true minimum on the adiabatic potential energy surface (solid red).

can be gained by considering which JT arrangements are absent from the list of stable minima. As shown in Table S7 in the SI, based on the already established rules, there should be two more stable JT arrangements-H4z44 and Oz444. However, multiple attempts to optimize these minima failed and always lead to proton transfer (to the O4z44 and $\mathrm{Hz} 444$ minima). This strongly suggests that JT axes towards the hydroxide ligand entail an additional energetic cost, similar to the JT axes towards a vanadate ligand. However, our results do not allow quantifying the energetic cost of a hydroxide JT axis, as none of the optimized minima contained one. Hence, we can only conjecture a fifth rule, saying that hydroxide JT axes are energetically unfavorable. This rule could be explained by the fact that the ligand involved in a JT axis obtains a rather high electron density through the filled $d_{z^{2}}$ orbital, and this higher electron density makes the ligand more basic such that it is energetically more favorable to move the proton to the JT axis.

In Mn3344 ( $\left.S_{1}\right)$, we find two low-energy minima, O4zx4 and O44xy. Both are analogous to the two-axes crossing minimum (44xy) that is the lowest-energy minimum of the precatalyst in the same oxidation state. The other five stable minima obtained for the catalyst in the Mn3344 oxidation state (Hx4x4, Oy4x4, Oxz44, Hy4x4, Hz4x4) all feature an apical JT axis. This can be identified as the reason for the slightly higher energy of these five minima $(2.0-4.2 \mathrm{kcal} / \mathrm{mol})$. Given the cost of an apical JT axis of $7.5 \mathrm{kcal} / \mathrm{mol}$, we estimate that the cost of a two-axes crossing in the catalyst is about 
$5 \mathrm{kcal} / \mathrm{mol}$. Based on these costs, Table S7 shows that there should be seven low-energy JT arrangements (4zx4, 44xy, $\mathrm{xz} 44, \mathrm{z} 4 \mathrm{x} 4, \mathrm{y} 44 \mathrm{x} 4, \mathrm{x} 4 \mathrm{x} 4, \mathrm{zz} 44)$ that should give rise to 14 low-lying minima for the Mn3344 oxidation state of the catalyst. Five of those would contain a JT axis towards the hydroxide ligand and are energetically unfavorable. From the remaining 9 minima, only seven were found and two minima (H44xy and Ox4x4) could not be optimized, even though none of the five already established rules exclude them. Based on the results of the optimizations, it appears that the barriers for conversion to a lower-energy minimum (O44xy and Oy4x4, respectively) are so small that the distinct minima vanish. This can be understood from Figure 5, where two diabatic states with constant electronic coupling produce two distinct minima if the minima have similar energies ( $a$ and b), but if one of the diabatic curves is shifted to too high energy (or the coupling is increased), only one minimum is obtained (c) for the ground state adiabatic surface.

For Mn3334 $\left(S_{0}\right)$, there are in principle 12 possible minima that do not involve the unfavorable vanadate JT axes (Figures S3 and S4). Out of those, we could optimize four minima, three of which have a two-axes crossing and an apical JT axis, and one minimum (O4zxy) that has a three-axis crossing. Rule five is followed in all cases, i.e., no minimum with a hydroxide JT axis was found (compare Table S8). In close analogy to Mn3344, two further minima that are not excluded by any of the proposed rules (Hy4xy and Oxzx4) could not be optimized due to vanishing barriers to lower-energy minima (Oy4xy and Oyzx4, respectively).

Lastly, for Mn3333 all optimizations converged to the same structure, Oyzxy. This structure exhibits a $\mathrm{Mn}-\mathrm{OH}_{2}$ bond length of about $3.7 \AA$, clearly showing signs of ligand dissociation, similar to the observation for the precatalyst in the Mn3333 oxidation state. Although a frequency calculation indicates that with the employed computational parameters this is indeed a minimum on the potential energy surface, it is doubtful whether this distant water ligand would stay associated with the complex in a real solvent environment. Hence, here we prefer to not discuss the Mn3333 structures in more detail.

As no structures with vanadate JT axes were optimized for Mn3334 and Mn3333 due to their large number, we cannot exclude the existance of other minima for these oxidation states. However, based on the findings of the precatalyst, we do not expect that any of those will actually constitute the lowest-energy minimum for these oxidation states.

To conclude, here we summarize the five heuristic rules about energetically unfavorable JT arrangements in the investigated compounds:

1. A JT axis at the apical $\mathrm{Mn}$ atom entails a $10 \mathrm{kcal} / \mathrm{mol}(7.5 \mathrm{kcal} / \mathrm{mol}$ in the catalyst $)$ cost.

2. A two-axes crossing costs $6 \mathrm{kcal} / \mathrm{mol}$ ( $5 \mathrm{kcal} / \mathrm{mol}$ in the catalyst).

3. A vanadate JT axis costs $12 \mathrm{kcal} / \mathrm{mol}$ (unknown but possibly similar in the catalyst).

4. A three-axes crossing costs $19 \mathrm{kcal} / \mathrm{mol}$ (roughly $17 \mathrm{kcal} / \mathrm{mol}$ in the catalyst).

5. A hydroxide JT axis in the catalyst costs a large amount of energy.

Other specifics of the JT arrangement-like the presence or absence of parallel, antiparallel, or skew arrangements or the position of the water proton-seem to play rather minor roles in the overall energetics.

These findings provide much needed guidance to explore the catalytic cycle of the MnV WOC and related compounds. The heuristic rules could be used to predict the energetically most favorable JT axis arrangements of intermediates and transition states in the mechanism, possibly allowing to exclude a large number of hypothetical structures without performing any quantum chemistry calculations. The finding about water dissociation from Mn3333 might imply that during the catalytic cycle the compound must first be oxidized before it can bind new water molecules. More generally, the rules can help explain the catalytic efficiency and guide the design of new catalysts. For example, assuming that an $\mathrm{Mn}_{4} \mathrm{O}_{4}$ cubane catalyst should support oxidation states from $\left[\mathrm{Mn}_{4}^{\mathrm{IV}}\right]$ to $\left[\mathrm{Mn}_{4}^{\mathrm{III}}\right]$, our rules predict that each $\mathrm{Mn}$ atom is required to have at least one coordination axis that can form a low-cost JT axis. This means that it might not be worthwhile to design a catalyst that is more stabilized by a larger vanadate ligand-if such a vanadate would coordinate with three $\mathrm{O}$ atoms on one $\mathrm{Mn}$ atom, then that $\mathrm{Mn}$ atom could not be reduced to $\mathrm{Mn}{ }^{\mathrm{III}}$ without forming an energetically costly vanadate JT axis. Hence, the existing MnV WOC seems to be a good compromise between chemical stabilization from the vanadate and chemical reactivity due to the weak bonds to the acetate ligands. 
(a) experimental

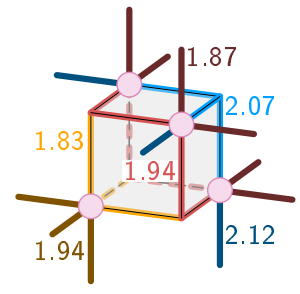

(b) computational

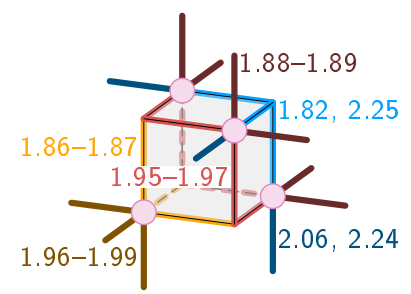

Figure 6: Comparison of experimental (a) and computed (b) bond lengths of the precatalyst in the Mn3344 oxidation state $\left(S_{1}\right)$. All lengths are given in $\AA$. The experimental structure is reported to be $C_{3 v}$ symmetric, whereas the computed structure shows JT distortions that are indicated by giving multiple values.

\subsection{Comparison to $\mathrm{X}$-ray structure}

At this point, a comparison of our findings with the X-ray crystallographic results of Schwarz et al. ${ }^{[8]}$ is on order. These authors reported the structure of the precatalyst in the Mn3344 oxidation state $\left(S_{1}\right)$-in the form of $(n$ $\left.\mathrm{Bu}_{4} \mathrm{~N}\right)_{3}\left[\mathrm{Mn}_{4} \mathrm{O}_{4}\left(\mathrm{~V}_{4} \mathrm{O}_{13}\right)(\mathrm{OAc})_{3}\right] \cdot 3 \mathrm{H}_{2} \mathrm{O}(\mathrm{CCDC} 898055)$-to be of ideal $C_{3 v}$ symmetry. The bond lengths found in the crystal structure are shown in Figure 6a. The lengths within the cubane structure differ significantly-the bonds with the apical Mn are the shortest ones (1.83 $\AA$ ), the bonds with the apical O are the longest ones $(2.07 \AA)$, and the other bonds are in between (1.94 $\AA$ ). The authors argue that the stretched bonds to the apical $\mathrm{O}$ atom are due to an attractive electrostatic interaction of that $\mathrm{O}$ atom with the vanadate ligand. The crystal structure data also shows that the Mn-O bonds towards the vanadate ligand are short $(1.87 \AA)$ and, interestingly, the ones towards the acetates are short at the apical Mn (1.94 $\AA$ ) and long at the other Mn atoms (2.12 $\AA$ ). No explanation is given for the disparity of the latter bond lengths.

Our results (Figure 6b) mostly agree with the experimental values. Bonds not involved in JT axes are $0.01-0.03 \AA$ longer than in the crystal structure, which could be either due to crystal packing/crystal environment effects that are not modeled in the computations, or due to a slight overestimation of the bond length by the employed level of theory. More importantly, while the experimental structure appears to be $C_{3 v}$ symmetric, our optimized structure exhibits a strong JT effect of the acetate axes of two of the non-apical Mn atoms. The corresponding bond lengths of the $\mathrm{Mn}^{\mathrm{IV}}$ atom are 1.82 and $2.06 \AA$, whereas the two $\mathrm{Mn}^{\mathrm{III}}$ atoms show values of 2.25 and $2.24 \AA$. In the computed results, the JT effect provides a clear explanation for the fact that the bonds from non-apical Mn to acetate are much longer than the ones from the apical Mn to the acetates.

One possible explanation for the discrepancy between the valence-delocalized crystal structure and valence-localized computational structure is dynamic disorder. ${ }^{[41]}$ This effect describes that in a crystal thermal motion allows the population of different equilibrium positions at different times or in different molecules. An argument for this explanation is the fact that the experimental bond lengths of the non-apical acetate axes (bond lengths 2.07 and $2.12 \AA$ ) are close to the average of the computed bond lengths (2.11 and 2.19 $\AA$ ), although again the computed bond lengths are slightly too long. However, dynamic disorder can only occur if the conversion barriers between the different JT arrangements are small enough compared to the temperature at which the crystal structure is recorded. For this reason, we computed all possible interconversion barriers between the stable JT arrangements of the precatalyst in the Mn3344 oxidation state. The 39 possible paths and the eight classes of symmetry-inequivalent paths are shown in Figure S5 in the SI, while Figure 7 presents potential energy surface scans for all eight classes. Based on the energetics, the most relevant interconversion path is the one that connects the lowest-energy minima of this structure (i.e., two different two-axes crossing minima, 44xy, 4zx4, and 4z4y). We find a potential energy barrier of only $3 \mathrm{kcal} / \mathrm{mol}$, which according to Eyring's equation corresponds to a life time of each JT arrangement of only $25 \mathrm{ps}$ at $300 \mathrm{~K}$. At the temperature used to record the crystal structure, ${ }^{8]} 150 \mathrm{~K}$, the life time is still only about $7.5 \mathrm{~ns}$, much shorter than the data collection time.

Hence, our computations strongly suggest that the MnV WOC undergoes very rapid conversion between localized minima with different JT arrangements. In the widely used classification scheme for mixed-valence transition metal complexes by Robin and Day, ${ }^{[42,43]}$ this corresponds to a class II intermediate localized situation. As has been noted 


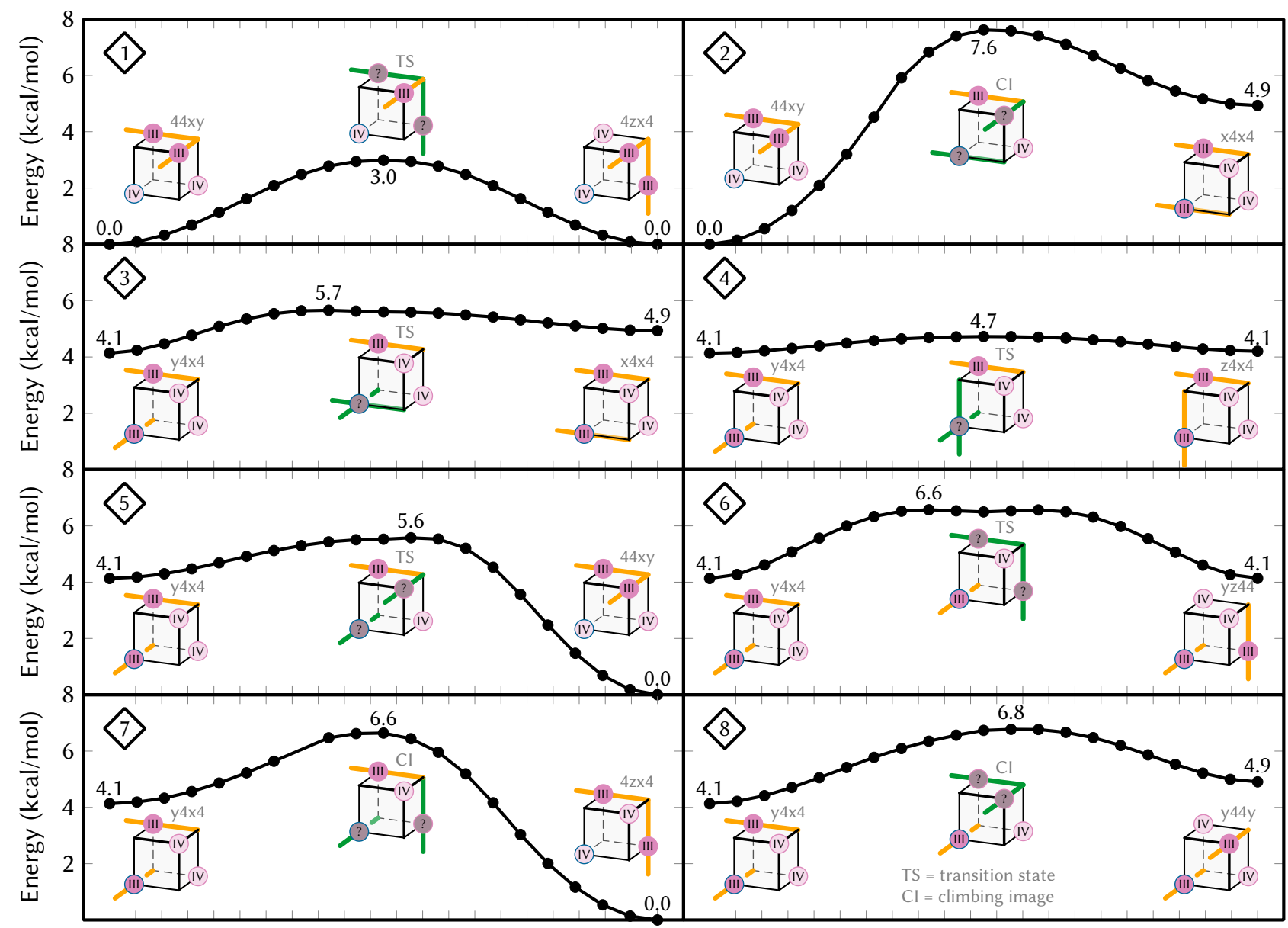

Reaction coordinate

Reaction coordinate

Figure 7: Potential energy surface scans of the eight symmetry-inequivalent transition paths of the precatalyst (Mn3344) (see also Figure S5), with energies given in $\mathrm{kcal} / \mathrm{mol}$. The drawings indicate the arrangement of the JT axes, with green color denoting the pair of JT axes that contract/expand along the transition path. Note that the scans were performed with linear interpolation in Cartesian coordinates and that some transition states could not be fully optimized. Hence, all barriers in the figure should be regarded as upper bounds to the true transition state energies. These calculations were performed with ORCA, explaining the small differences in the relative energies of the minima compared to Table 2.

before, ${ }^{[44]}$ computational results on such systems require a fine balance between dynamic and static correlation effects. Here, we use a GGA functional (BP86), which tends to suffer somewhat from self-interaction errors and thus should produce a too delocalized electronic structure. This is interesting, because for the MnV WOC we find an electronic structure that is more localized than the experiment even with a functional that should produce too much delocalization. Hence, we assume that the disagreement between experiment and computations is not due to the limitations of our level of theory. Instead, an explanation could be the different effect of the surroundings-in solution a localized electronic structure is formed, whereas in the crystal a delocalized one arises. Nevertheless, we suggest that it might be worthwhile to revisit the delocalization-localization issue in this complex (optimally for multiple oxidation states) from the experimental side.

The very low interconversion barriers that we found in our work for the solvated MnV WOC can also help to explain the distinguishing feature of the optimal ligand exchange pathway found for the catalyst activation mechanism. ${ }^{[25]}$ As shown above, a JT axis of $\mathrm{Mn}^{\mathrm{III}}$ tends to be weaker and more easily undergoes ligand dissociation than a coordination axis of $\mathrm{Mn}^{\mathrm{IV}}$. The low barriers in Figure 7 can then be exploited to easily switch the location of the most reactive coordination site on the cubane-like first weakening a bond to the acetate ligand, exchanging this ligand with water from the solution, then moving the JT axis to another Mn center and exchanging a second ligand. ${ }^{[25]}$ 


\section{Conclusion}

We reported a comprehensive computational study of the Jahn-Teller effect on the energetics of different oxidation and tautomeric states of the manganese-oxo vanadate catalyst $\left[\left(\mathrm{Mn}_{4} \mathrm{O}_{4}\right)\left(\mathrm{V}_{4} \mathrm{O}_{13}\right)(\mathrm{OAc})_{3}\right]^{n-}$ and its activated species $\left[\left(\mathrm{Mn}_{4} \mathrm{O}_{4}\right)\left(\mathrm{V}_{4} \mathrm{O}_{13}\right)(\mathrm{OAc})_{2}\left(\mathrm{H}_{2}\right)(\mathrm{OH})\right]^{n-}$. To this end, we performed structure optimizations of all combinatorially possible arrangements of the Jahn-Teller axes at the $\mathrm{Mn}^{\mathrm{III}}$ atoms. We found that for each oxidation state, only few Jahn-Teller arrangements are energetically stable such that one can expect only one or two arrangements for each oxidation state at thermal equilibrium.

By correlating the energetics of the different optimized minima with the main structural features, we obtained a set of heuristic rules that can be used to judge the relative energies of different Jahn-Teller arrangements. The first rule states that a Jahn-Teller axis at the apical $\mathrm{Mn}$ atom, the one bonded to all three acetate ligands, is energetically less favorable (by about $10 \mathrm{kcal} / \mathrm{mol}$ ) than a Jahn-Teller axis at any of the three other atoms. Rule two states that the crossing of two Jahn-Teller axes brings an energetic cost (about $6 \mathrm{kcal} / \mathrm{mol}$ ). Third, Jahn-Teller axes pointing towards the vanadate $\mathrm{O}$ atoms are very unfavorable (about $12 \mathrm{kcal} / \mathrm{mol}$ ). The fourth rule associates a very high cost (about $19 \mathrm{kcal} / \mathrm{mol}$ ) to the crossing of three Jahn-Teller axes. Finally, in the activated complex, also Jahn-Teller axes towards the hydroxide ligand entail a high energetic cost. Although the precise energetic costs will surely depend on the molecule (and will also vary depending on the level of theory), these rules can help to understand and predict the structure of transition metal oxo cubanes (and other clusters) in general, as well as explain their reactivity and properties. As discussed above, the availability of different Jahn-Teller minima and the involved low barriers for interconversion between these minima can facilitate important reactions for oxygen-evolving catalysts, such as the ligand exchange observed during activation of the MnV WOC. Thus, we hope that the presented findings will enable the directed search for even more efficient catalysts.

\section{Acknowledgement}

S.M. and L.G. gratefully acknowledge funding from the Austrian Science Fund (FWF) within the project I3987 ("Catalight") and from the Deutsche Forschungsgemeinschaft (DFG) within project 364549901 (TRR 234 "CataLight"). The University of Vienna is gratefully acknowledged for continuous support and the Vienna Scientific Cluster (VSC3) for generous allocation of computational resources.

\section{Supporting Information}

Depictions and lists of all possible JT arrangements and their symmetry relations. Depictions of optimized minima. Bond lengths from pre-optimizations and full optimizations. Predicted relative energies of all possible JT arrangements based on the five presented rules. Overview over JT conversion pathways. Cartesian coordinates (xyz format) for all stable minima.

\section{Keywords}

Density functional calculations $\cdot$ Jahn-Teller distortion $\cdot$ Polyoxometalates $\cdot$ Reaction mechanisms $\cdot$ Water splitting 


\section{Table of Contents Figure}

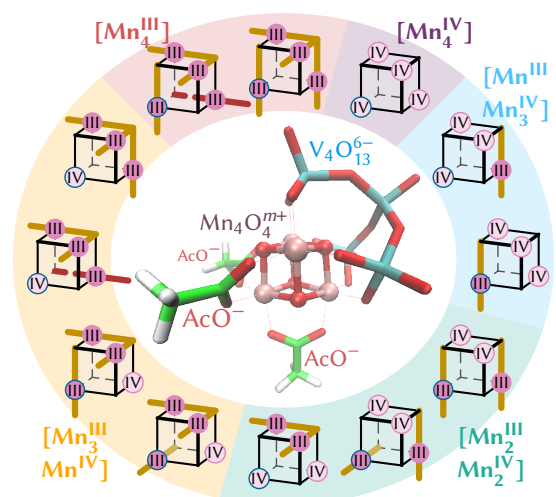

Jahn-Teller effects in the manganese-oxo vanadate catalyst $\left[\left(\mathrm{Mn}_{4} \mathrm{O}_{4}\right)\left(\mathrm{V}_{4} \mathrm{O}_{13}\right)(\mathrm{OAc})_{3}\right]^{n-}$ and its activated form $\left[\left(\mathrm{Mn}_{4} \mathrm{O}_{4}\right)\left(\mathrm{V}_{4} \mathrm{O}_{13}\right)(\mathrm{OAc})_{2}\left(\mathrm{H}_{2}\right)(\mathrm{OH})\right]^{n-}$ lead to a many local minima for different oxidation states and enhance the catalyst's reactivity.

\section{References}

[1] S. Berardi, S. Drouet, L. Francàs, C. Gimbert-Suriñach, M. Guttentag, C. Richmond, T. Stoll, A. Llobet, Chem. Soc. Rev. 2014, 43, 7501-7519.

[2] J. D. Blakemore, R. H. Crabtree, G. W. Brudvig, Chem. Rev. 2015, 115, 12974-13005.

[3] I. Roger, M. A. Shipman, M. D. Symes, Nat. Rev. Chem. 2017, 1, 0003.

[4] R. Matheu, P. Garrido-Barros, M. Gil-Sepulcre, M. Z. Ertem, X. Sala, C. Gimbert-Suriñach, A. Llobet, Nat. Rev. Chem. 2019, 3, 331-341.

[5] W. F. Ruettinger, C. Campana, G. C. Dismukes, J. Am. Chem. Soc. 1997, 119, 6670-6671.

[6] J.-Z. Wu, E. Sellitto, G. P. A. Yap, J. Sheats, G. C. Dismukes, Inorg. Chem. 2004, 43, 5795-5797.

[7] R. Brimblecombe, G. Swiegers, G. Dismukes, L. Spiccia, Angew. Chem. Int. Ed. 2008, 47, 7335-7338.

[8] B. Schwarz, J. Forster, M. K. Goetz, D. Yücel, C. Berger, T. Jacob, C. Streb, Angew. Chem. Int. Ed. 2016, 55, 6329-6333.

[9] H. B. Lee, A. A. Shiau, P. H. Oyala, D. A. Marchiori, S. Gul, R. Chatterjee, J. Yano, R. D. Britt, T. Agapie, J. Am. Chem. Soc. 2018, 140, 17175-17187.

[10] B. Kok, B. Forbush, M. Mcgloin, Photochem. Photobiol. 1970, 11, 457-475.

[11] P. E. Siegbahn, Physical Chemistry Chemical Physics 2013, 1827, 1003-1019.

[12] N. Cox, M. Retegan, F. Neese, D. A. Pantazis, A. Boussac, W. Lubitz, Science 2014, 345, 804-808.

[13] V. Krewald, M. Retegan, N. Cox, J. Messinger, W. Lubitz, S. DeBeer, F. Neese, D. A. Pantazis, Chem. Sci. 2015, 6, 1676-1695.

[14] W. F. Ruettinger, D. M. Ho, G. C. Dismukes, Inorg. Chem. 1999, 38, 1036-1037.

[15] W. Ruettinger, M. Yagi, K. Wolf, S. Bernasek, G. C. Dismukes, F. Am. Chem. Soc. 2000, 122, 10353-10357.

[16] R. Brimblecombe, A. M. Bond, G. C. Dismukes, G. F. Swiegers, L. Spiccia, Phys. Chem. Chem. Phys. 2009, 11, 6441. 
[17] G. C. Dismukes, R. Brimblecombe, G. A. N. Felton, R. S. Pryadun, J. E. Sheats, L. Spiccia, G. F. Swiegers, Acc. Chem. Res. 2009, 42, 1935-1943.

[18] N. E. Chakov, K. A. Abboud, L. N. Zakharov, A. L. Rheingold, D. N. Hendrickson, G. Christou, Polyhedron 2003, 22, 1759-1763.

[19] N. E. Chakov, A. E. Thuijs, W. Wernsdorfer, A. L. Rheingold, K. A. Abboud, G. Christou, Inorg. Chem. 2016, 55, 8468-8477.

[20] J. S. Kanady, E. Y. Tsui, M. W. Day, T. Agapie, Science 2011, 333, 733-736.

[21] J. S. Kanady, J. L. Mendoza-Cortes, E. Y. Tsui, R. J. Nielsen, W. A. Goddard, T. Agapie, f. Am. Chem. Soc. 2013, 135, 1073-1082.

[22] C. E. Dubé, D. W. Wright, S. Pal, P. J. Bonitatebus, W. H. Armstrong, J. Am. Chem. Soc. 1998, 120, 3704-3716.

[23] S. Mukhopadhyay, S. K. Mandal, S. Bhaduri, W. H. Armstrong, Chem. Rev. 2004, 104, 3981-4026.

[24] F. L. Huber, S. Amthor, B. S. B. Mizaikoff, C. Streb, S. Rau, Sustain. Energy Fuels 2018, 2, 1974-1978.

[25] G. Cárdenas, I. Trentin, L. Schwiedrzik, D. Hernández-Castillo, G. A. Lowe, J. Kund, C. Kranz, S. Klingler, R. Stach, B. Mizaikoff, P. Marquetand, J. J. Nogueira, C. Streb, L. González, ChemRxiv 2021.

[26] F. Neese, WIREs Comput. Mol. Sci. 2012, 2, 73-78.

[27] F. Neese, WIREs Comput. Mol. Sci. 2017, 8, e1327.

[28] J. P. Perdew, Phys. Rev. B 1986, 33, 8822-8824.

[29] A. D. Becke, Phys. Rev. A 1988, 38, 3098-3100.

[30] F. Weigend, R. Ahlrichs, Phys. Chem. Chem. Phys. 2005, 7, 3297-3305.

[31] D. A. Pantazis, X.-Y. Chen, C. R. Landis, F. Neese, J. Chem. Theory Comput. 2008, 4, 908-919.

[32] E. van Lenthe, E. J. Baerends, J. G. Snijders, f. Chem. Phys. 1993, 99, 4597-4610.

[33] E. van Lenthe, E. J. Baerends, J. G. Snijders, f. Chem. Phys. 1994, 101, 9783-9792.

[34] S. Grimme, WIREs Comp. Mol. Sci. 2011, 1, 211-228.

[35] V. Barone, M. Cossi, f. Phys. Chem. A 1998, 102, 1995-2001.

[36] M. J. Frisch, G. W. Trucks, H. B. Schlegel, G. E. Scuseria, M. A. Robb, J. R. Cheeseman, G. Scalmani, V. Barone, G. A. Petersson, H. Nakatsuji, X. Li, M. Caricato, A. V. Marenich, J. Bloino, B. G. Janesko, R. Gomperts, B. Mennucci, H. P. Hratchian, J. V. Ortiz, A. F. Izmaylov, J. L. Sonnenberg, D. Williams-Young, F. Ding, F. Lipparini, F. Egidi, J. Goings, B. Peng, A. Petrone, T. Henderson, D. Ranasinghe, V. G. Zakrzewski, J. Gao, N. Rega, G. Zheng, W. Liang, M. Hada, M. Ehara, K. Toyota, R. Fukuda, J. Hasegawa, M. Ishida, T. Nakajima, Y. Honda, O. Kitao, H. Nakai, T. Vreven, K. Throssell, J. A. Montgomery, Jr., J. E. Peralta, F. Ogliaro, M. J. Bearpark, J. J. Heyd, E. N. Brothers, K. N. Kudin, V. N. Staroverov, T. A. Keith, R. Kobayashi, J. Normand, K. Raghavachari, A. P. Rendell, J. C. Burant, S. S. Iyengar, J. Tomasi, M. Cossi, J. M. Millam, M. Klene, C. Adamo, R. Cammi, J. W. Ochterski, R. L. Martin, K. Morokuma, O. Farkas, J. B. Foresman, D. J. Fox, Gaussian16 Revision A.03 2016, gaussian Inc. Wallingford CT.

[37] S. Grimme, J. Antony, S. Ehrlich, H. Krieg, f. Chem. Phys. 2010, 132, 154104.

[38] J. Tomasi, B. Mennucci, R. Cammi, Chem. Rev. 2005, 105, 2999-3094.

[39] P. E. M. Siegbahn, Chem. Eur. F. 2006, 12, 9217-9227. 
[40] V. Ásgeirsson, B. Birgisson, R. Bjornsson, U. Becker, C. Riplinger, F. Neese, H. Jónsson, f. Chem. Theory Comput. 2021, accepted.

[41] H. B. Bürgi, Annu. Rev. Phys. Chem. 2000, 51, 275-296.

[42] M. B. Robin, P. Day, in Advances in Inorganic Chemistry and Radiochemistry, volume 10, chapter 3, Elsevier 1968, $247-422$.

[43] K. D. Demadis, C. M. Hartshorn, T. J. Meyer, Chem. Rev. 2001, 101, 2655-2686.

[44] M. Parthey, M. Kaupp, Chem. Soc. Rev. 2014, 43, 5067-5088. 